\title{
A Cross Sectional Study On Quality Of Sleep Among Adult Population In An Urban Area Of Pahang
}

Hafizah binti Pasi ${ }^{1}$, Razman Mohd Rus ${ }^{1}$, Karimah Hanim Abd Aziz ${ }^{1}$, Muhammad Zubir Yusof $^{1}$, Noor Artika Hassan ${ }^{1}$

${ }^{1}$ Department of Community Medicine, Kulliyyah of Medicine International Islamic University Malaysia

Presenter: Hafizah Pasi

Introduction: Sleep is one of the important needs in human life and good quality of sleep among adult population reduced the risk factor for adverse health outcome. This study aimed to determine the quality of sleep and its related factors among adult population in an urban area of Pahang. Materials and Methods: Cross sectional study was done on a selected urban housing area in Pahang. Participants were adult population, aged more than 18 years old (observation unit) in selected houses $(n=180)$. Measurements obtained via the questionnaire include respondents' socio-demographic, co-morbidity, smoking status, physical activity, monthly household income and caffeinated drinks consumed. Sleep quality was measured using the validated Pittsburgh Sleep Quality Index (PSQI). Results: A total of 145 respondents (response rate 81\%) were available for data analysis. The prevalence of good sleeper (60\%) was more than poor sleeper. Adjusted logistic regression showed that only those who did not take caffeinated drinks in the past one week had higher chance of having good quality of sleep as compared to those who take $(O R=3.6, p=0.048)$. No other significant relationship was seen between quality of sleep and socio-demographic characteristics, exercise or co-morbid history. Conclusion: Majority of adult population in this selected urban area in Pahang have good quality of sleep and result showed significant relationship between quality of sleep with not taking caffeinated drinks, but not other factors. Nevertheless, programs such as sleep quality awareness campaign need to be done to educate the population and maintain this good sleep quality. 